\title{
Implementing phase-covariant cloning in circuit quantum electrodynamics
}

\author{
Meng-Zheng Zhu ${ }^{\mathrm{a}, \mathrm{b}}$, Liu Ye $\mathrm{Ya}^{\mathrm{a}, *}$ \\ ${ }^{a}$ School of Physics and Material Science, Anhui University, Hefei 230039, China \\ ${ }^{b}$ School of Physics and Electronic Information, Huaibei Normal University, Huaibei 235000, \\ China
}

\begin{abstract}
An efficient scheme is proposed to implement phase-covariant quantum cloning by using a superconducting transmon qubit coupled to a microwave cavity resonator in the strong dispersive limit of circuit quantum electrodynamics (QED). By solving the master equation numerically, we plot the Wigner function and Poisson distribution of the cavity mode after each operation in the cloning transformation sequence according to two logic circuits proposed. The visualizations of the quasi-probability distribution in phase-space for the cavity mode and the occupation probability distribution in the Fock basis enable us penetrate the evolution process of cavity mode during the phase-covariant cloning (PCC) transformation. With the help of numerical simulation method, we find out that the present cloning machine is not the isotropic model because its output fidelity depends on the polar angle and the azimuthal angle of the initial input state on the Bloch sphere. The fidelity for the actual output clone of the present scheme is slightly smaller than one in the theoretical case. The simulation results are consistent with the theoretical ones. This further corroborates our scheme based on circuit QED can implement efficiently PCC transformation.

Keywords: , Quantum information, Quantum cryptography and communication security, quantum cloning machine
\end{abstract}

\footnotetext{
* Corresponding author

Email address: yeliu@ahu.edu.cn (Liu Ye)
}

Preprint submitted to Annals of Physics

June 21, 2016

(C) 2016. This manuscript version is made available under the Elsevier user license http://www.elsevier.com/open-access/userlicense/1.0/ 


\section{Introduction}

The basic principles of quantum mechanics protect the quantum information from exposure to illegal persons. As mentioned in the no-cloning theorem [1], perfect quantum cloning of arbitrary unknown quantum state is forbidden

5 because of the inherent property of quantum mechanics. This theorem is a consequence of the linearity of quantum mechanics and it is at the basis of the security of quantum communication protocols including quantum key distribution. Quantum key distribution (QKD) is one of the well-known topics of quantum information [2] due to the practical application to quantum communication. However, along with the rapid development of quantum information theory the unknown quantum state can be copied approximately with maximum fidelity and with the copying quality to being independent of initial input state because the no-cloning theorem does not forbid imperfect cloning. The most notable example is the universal quantum cloning machine (UQCM) proposed by Bužek and Hillery [3] in 1996. For the investigation of approximate quantum cloning, it is one of the reasons that from a fundamental research point of view the optimal quantum clones provide insight into the fundamental limits on the manipulation and distribution of quantum information. The other more practical reason is that these clones can be used as very efficient eavesdropping attacks on quantum key distribution protocols [4]. And a great of effort $[5,6,7,8,9,10,11,12,13]$ has been devoted to the realization of the optimal approximations to quantum cloning machines (QCMs) motivated by the seminal scheme [3]. Recently, some experiments on quantum cloning have been reported $[14,15,16,17,18,19,20,21]$. In 2014, a review about various QCMs has been reported by Fan et al. [22]. Bužek and Hillery [3] designed the UQCM which produces two approximate copies from an unknown pure qubit state. The UQCM is a state-independent symmetric cloning. That is to say, all qubit states are cloned with the same fidelity $F=5 / 6$.

It is often the case that we possess some a priori information about the quantum state but we do not know it exactly. That is why it is important 
for the study of state-independent cloning. For example, all existing quantum cryptographic experiments are using quantum states that are on the equator, rather than states that span the whole Bloch sphere. Motivated by such a physical implementation of quantum communication ideas, Bruß et.al [6] have presented a PCC with the fidelity 0.854 for the restriction of the input set to the equator of the Bloch sphere. The qubit to be copied lies on the equator, that is to say, the azimuthal angle $\phi$ on the Bloch sphere can be arbitrary while the polar angle fixed $\theta=\pi / 2$ for the qubit. The optimal cloning of equatorial qubits represents an efficient eavesdropping attack on the BB84 quantum key distribution protocol [23]. Using the available priori information about the original state to be cloned, it allows us to optimize the cloning process which performs better cloning than the UQCM. Fiurášek [24, 25] has generalized the PCC with the prior knowledge of polar angle $\theta$ from the full range $[0, \pi]$ and the azimuthal angle $\phi$ completely unknown, i.e., uniformly distributed between $45[0,2 \pi]$. Two optimal 1-to-2 symmetric PCC schemes have been provided $[24,25]$ : one for the states to be copied in the upper hemisphere of the Bloch sphere and the other for those in the lower. Fan et al. [26] have extended the PCC for qubit to one input to many outputs.

We present an efficient scheme for implementation of cloning of qubit states with a priori known value of the polar angle by using a superconducting transmon qubit coupled to a waveguide cavity resonator with a highly ideal off-resonant coupling. The information of initial qubit state would be cloned approximatively onto a resonator within a higher-dimensional Hilbert space in a cavity, except for onto the transmon qubit by itself. Several proposals exist to use this larger Hilbert space for redundant encoding to allow quantum error correction [27]. Copying an effective two-level system into the large Hilbert space of a continuous variable system, such as that of a harmonic oscillator, may even be more advantageous by allowing for more compact information processing. Recently a proposal [28] has been put forward for encoding, manipulating, and protecting information in photon number parity eigenstates of microwaves dispersively coupled to a superconducting qubit. This provides an 
exciting new development in the field of coherent state quantum computing. Here, using a three-dimensional circuit QED architecture, we realized a highly ideal strong-dispersive coupling, where the strengths of the off-resonant qubitcavity interactions were several orders of magnitude greater than the cavity decay rate and higher-order nonlinearities. The strong coupling and coherence possible in this new architecture, along with a new protocol for efficiently transferring qubit states to coherent light states [28], have now enabled Vlastakis et al. [29] to create Schrödinger cats of an impressive size, consisting of up to 111 microwave photons trapped inside the 3D cavity. In addition, cavity resonators have proven a valuable resource for a quantum memory to store and shuttle information $[30,31]$ for superconducting quantum circuits.

\section{Protocol for implementing state-denpendent cloning machine}

The circuit QED system we consider, called a three-dimensional superconducting transmon $[29,32]$, contains a Josephson junction antenna, dipole coupled to the quantized electric field of a microwave resonator. The sample setup is described in detail in Ref. [32, 33]. For the purposes of the cloning scheme discussed here, the off-resonant coupling of the transmon qubit and the microwave resonator, in the strong dispersive limit of circuit QED, can be well described so by the Hamiltonian

$$
H / \hbar=\omega_{c} \hat{a}^{\dagger} \hat{a}+\omega_{q}|e\rangle\left\langle e\left|-\chi \hat{a}^{\dagger} \hat{a}\right| e\right\rangle\langle e|,
$$

with $\omega_{c}$ and $\omega_{q}$ the cavity and qubit transition frequency, respectively, $\hat{a}$ the annihilation operator of a cavity excitation, $\chi$ the dispersive shift, and $|e\rangle$ the excited state of the qubit. Here the nonlinear Josephson oscillator is viewed approximatively as a two-level system, only to consider the lowest two energy 85 levels ( $|g\rangle$ and $|e\rangle)$ of the qubit. This interaction produces a state-dependent shift in either the qubit or cavity transition frequency, that is to say, in the strong dispersive regime $[28,34]$ where both the qubit and cavity transition frequencies split into well-resolved spectral lines indexed by the number of excitations in the transmon qubit and the microwave resonator. The qubit transition 

into $\omega_{q}^{n}=\omega_{q}-n \chi$ corresponding to the qubit frequency when the cavity is in the photon number state $|n\rangle$. The cavity frequency $\omega_{c}$ splits into $\omega_{c}^{g}$ and $\omega_{c}^{e}$ corresponding to the cavitys frequency when the qubit in the cavity is in the ground state $|g\rangle$ or the excited state $|e\rangle$. Recent experiment by Paik et al. [32] has demonstrated that the dispersive shift $\chi$ is about three orders of magnitude larger than both the qubit and cavity transition linewidths. This spectral resolution can ensure that we should implement selective rotation operation of the transmon qubit conditioned on the photon number in the cavity and selective phase shift operation of the cavity field conditioned on the qubit state $[29,33,35]$.

By using a superconducting transmon qubit coupled to a microwave cavity resonator in the strong dispersive limit, we demonstrated an efficient scheme for implementing PCC for the input qubit states with a priori known expectation value of the Pauli operator $\sigma_{z}$. The quantum information to be cloned is encoded in the superconducting transmon. The superconducting transmon qubit is in the state

$$
|\varphi\rangle_{q}^{(i n)}=\cos \frac{\theta}{2}|e\rangle+e^{i \phi} \sin \frac{\theta}{2}|g\rangle
$$

If the expectation value $\left\langle\sigma_{z}\right\rangle>0$ for the quantum state to be cloned, we employ quantum logic circuit in the upper half of Fig. 1 to implement cloning transformation. If $\left\langle\sigma_{z}\right\rangle<0$, we employ quantum logic circuit in the lower half of Fig. 1. After cloning transformation, one cloner is the superconducting transmon while the other is the cavity mode. For the cavity mode, we consider the quasi-orthogonal computational basis $|-\alpha\rangle,|\alpha\rangle$. Because the scalar product of two coherent states is $\langle\beta \mid \alpha\rangle=\exp \left[-\frac{1}{2}\left(|\alpha|^{2}+|\beta|^{2}\right)+\alpha \beta^{*}\right]$, the non-orthogonality of the two coherent states $|\langle-\alpha \mid \alpha\rangle|^{2}=\exp \left[-4|\alpha|^{2}\right] \approx 8.3 \times 10^{-7}$ corresponding 115 to the mean number of photon $\bar{n}_{c}=|\alpha|^{2}=3.5$. If $|\alpha|$ is sufficiently large, the scalar product $\langle-\alpha \mid \alpha\rangle \approx 0$. In order to clone the state of the transmon qubit to the cavity mode, we prepare initially a cavity in the state $|-\alpha\rangle$. During the cloning transformation, we require two conditional operations: the cavity phase 


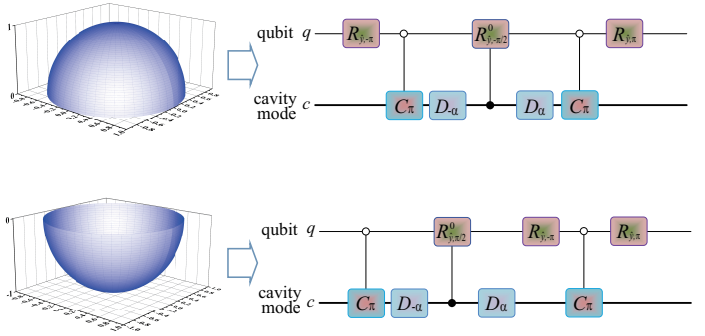

Figure 1: A Circuit diagram of phase covariant cloning machine based on selective rotation operation of the transmon qubit conditioned on the $|0\rangle$ cavity state, selective phase shift operation of the cavity mode conditioned on the excited state of transmon qubit, the unconditional cavity displacement, and the unconditional rotation operation of the transmon qubit. The input state of transmon qubit $q$ to be cloned is described by Eq. (2) while the cavity mode $c$ is initially in the coherent state $|-\alpha\rangle$. For the state of Eq. (2) in the northern hemisphere, we employ the upper logic circuit to implement cloning transformation while for the state in the southern hemisphere, we employ the lower logic circuit.

shift $[29,33]$ conditioned on the qubit state and the qubit rotation $[28,29]$ conditioned on the photon-number in the cavity. We combined these operations with unconditional qubit and cavity operations to efficiently implement cloning transformation. The conditional phase shift of the cavity mode can be described as

$$
C_{\chi t}=\exp \left[i \chi t \hat{a}^{\dagger} \hat{a}|e\rangle\langle e|\right]=\hat{I} \otimes|g\rangle\left\langle g\left|+e^{i \chi t \hat{a}^{\dagger} \hat{a}} \otimes\right| e\right\rangle\langle e| .
$$

This conditional phase shift $\chi t$ appears from the free evolution of the dispersive term in Hamiltonian Eq. (1) for a time $t$. For the present cloning scheme, the conditional phase shift $\chi t$ is equal to $\pi$, and $\hat{P} \equiv e^{i \pi \hat{a}^{\dagger} \hat{a}}$ is viewed as the photon number parity operator. Because the coherent state may be expanded in terms of the photon-number states as $|\alpha\rangle=e^{-|\alpha|^{2} / 2} \sum_{n=0} \frac{\alpha^{n}}{\sqrt{n !}}|n\rangle$, the operation of conditional phase shift enables us to copy the information of qubit state into the phases of many photons simultaneously in the cavity mode. This operation $C_{\pi}$ causes the cavity mode to acquire a phase shift $\pi$ conditional on the qubit state being exactly an odd number of photons in the cavity. The other conditional 
operation is a rotation of the transmon qubit by an angle $\theta_{0}$ around the y-axis of the Bloch sphere conditioned on the $|0\rangle$ cavity state, that is, the operation can be ideally given by [29]

$$
\begin{aligned}
& R_{\hat{y}, \theta_{0}}^{0}=|0\rangle\left\langle 0\left|\otimes R_{\hat{y}, \theta_{0}}+\sum_{n \neq 0}\right| n\right\rangle\langle n| \otimes \hat{I}, \\
& R_{\hat{y}, \theta_{0}}=e^{-i \frac{\theta_{0}}{2} \sigma_{y}} .
\end{aligned}
$$

In order to acquire the conditional qubit rotation, we can introduce a classical drive on resonance with the photon-number-dependent split peak $\omega_{q}^{0}$. The classical drive field is a Gaussian pulse which is used to selectively address $\omega_{q}^{0}$ without driving $\omega_{q}^{n>0}$. The amplitude envelope of classical drive is a time-dependent real value $\varepsilon(t)=A e^{-\sigma_{\omega}^{2} t^{2} / 2}$ where $\sigma_{\omega}$ is the spectral width of the Gaussian pulse. Taking the fidelity of the conditional rotation operation into account, we actually have a looser restriction onto the value of spectral width $\sigma_{\omega}=4|\alpha|^{2} \chi / 5$ as mentioned in the Supplementary Material of Ref. [29]. In order to perform a qubit $\pi / 2$ rotation on the $\omega_{q}^{0}$ transition, we assign $\sqrt{\pi / 8} \sigma_{\omega}$ to the amplitude $A$ of the drive required due to the rotation angle $\theta_{0}=2 \int \varepsilon(t) d t$. To perform cloning transformation, we have the ability to implement the unconditional rotation Eq. (4) on the transmon qubit which is independent of the cavity state, and to perform the standard cavity displacement $D_{\alpha}=\exp \left[\alpha \hat{a}^{\dagger}-\alpha^{*} \hat{a}\right]$ through a very short pulse which will displace the cavity state regardless of the qubit state. In the cloning scheme we solve numerically the master equation formalism involved with the Hamiltonian Eq. (1) accounting for the effects of photon loss using the QuTiP [36].

In the case of the polar angle fixed $\theta \in[0, \pi / 2]$, that is to say, the available a priori information is the expectation value $\left\langle\sigma_{z}\right\rangle>0$ for the input state to be cloned, the unitary transformation of state-dependent cloning can be written as

$$
|\varphi\rangle_{q}^{(\mathrm{in})}|-\alpha\rangle \rightarrow \cos \frac{\theta}{2}|e\rangle|-\alpha\rangle+\frac{1}{\sqrt{2}} e^{i \phi} \sin \frac{\theta}{2}(|e\rangle|\alpha\rangle+|g\rangle|-\alpha\rangle) .
$$

The reduced density operator of each output mode, such as cloners $q$ and $c$, 
after the cloning procedure, is given as:

$$
\begin{aligned}
& \rho_{q}^{\text {(out) }}=\frac{1}{4}(3+\cos \theta)|e\rangle\left\langle e\left|+\frac{1}{4}(1-\cos \theta)\right| g\right\rangle\langle g|+\left(\frac{1}{2 \sqrt{2}} e^{i \phi} \sin \theta|g\rangle\langle e|+\text { h.c. }\right), \\
& \rho_{c}^{\text {(out) }}=\frac{1}{4}(3+\cos \theta)|-\alpha\rangle\left\langle-\alpha\left|+\frac{1}{4}(1-\cos \theta)\right| \alpha\right\rangle\langle\alpha|+\left(\frac{1}{2 \sqrt{2}} e^{i \phi} \sin \theta|\alpha\rangle\langle-\alpha|+\text { h.c. }\right) .
\end{aligned}
$$

Due to the fidelity defined by $F_{q}=_{q}^{\text {(in) }}\left\langle\varphi\left|\rho_{q}^{(\text {out })}\right| \varphi\right\rangle_{q}^{(\text {in })}$, the fidelity of two clones for the symmetric cloning transformation can easily be calculated as

$$
F_{q}=F_{c}=\frac{1}{4}\left[\sqrt{2}(1+\sqrt{2})+\cos \theta+(1-\sqrt{2}) \cos ^{2} \theta\right] .
$$

In the other case of the polar angle fixed $\theta \in[\pi / 2, \pi]$, the unitary transformation of state-dependent cloning can be written as

$$
|\varphi\rangle_{q}^{(\mathrm{in})}|-\alpha\rangle \rightarrow \frac{1}{\sqrt{2}} \cos \frac{\theta}{2}(|e\rangle|\alpha\rangle+|g\rangle|-\alpha\rangle)+e^{i \phi} \sin \frac{\theta}{2}|g\rangle|\alpha\rangle .
$$

The reduced density operator of each output mode, such as cloners $q$ and $c$, after the cloning procedure, is given as:

$$
\begin{aligned}
& \rho_{q}^{\text {(out) }}=\frac{1}{4}(1+\cos \theta)|e\rangle\left\langle e\left|+\frac{1}{4}(3-\cos \theta)\right| g\right\rangle\langle g|+\left(\frac{1}{2 \sqrt{2}} e^{i \phi} \sin \theta|g\rangle\langle e|+\text { h.c. }\right), \\
& \rho_{c}^{\text {(out) }}=\frac{1}{4}(1+\cos \theta)|-\alpha\rangle\left\langle-\alpha\left|+\frac{1}{4}(3-\cos \theta)\right| \alpha\right\rangle\langle\alpha|+\left(\frac{1}{2 \sqrt{2}} e^{i \phi} \sin \theta|\alpha\rangle\langle-\alpha|+\text { h.c. }\right) .
\end{aligned}
$$

Thus, the fidelity of two clones can easily be calculated as

$$
F_{q}=F_{c}=\frac{1}{4}\left[\sqrt{2}(1+\sqrt{2})-\cos \theta+(1-\sqrt{2}) \cos ^{2} \theta\right] .
$$

The Wigner function is a representation of a quantum state in a continuous variable basis and is a quasi-probability distribution in phase space. It can be defined as $W(\alpha)=\frac{2}{\pi} \operatorname{Tr}\left[D_{\alpha}^{\dagger} \rho_{c} D_{\alpha} \hat{P}\right]$ where $\rho_{c}$ is the reduced density matrix of cavity state. The Wigner function of a cavity could be determined by measuring the mean photon parity $\langle\hat{P}\rangle$ at many points in the cavity phase space. Using this reduced density matrix of cavity mode, we calculate and depict the Wigner function to show the interference fringes, highlighting the quantum features of the cavity state. The numerical simulation could be done by solving a master equation with the decay of cavity. The corresponding parameters are $\bar{n}_{c}=3.5$, $\omega_{c} / 2 \pi=8.1794 \mathrm{GHz}, \omega_{q} / 2 \pi=7.4552 \mathrm{GHz}, \chi / 2 \pi=5 \mathrm{MHz}$, the cavity decay 

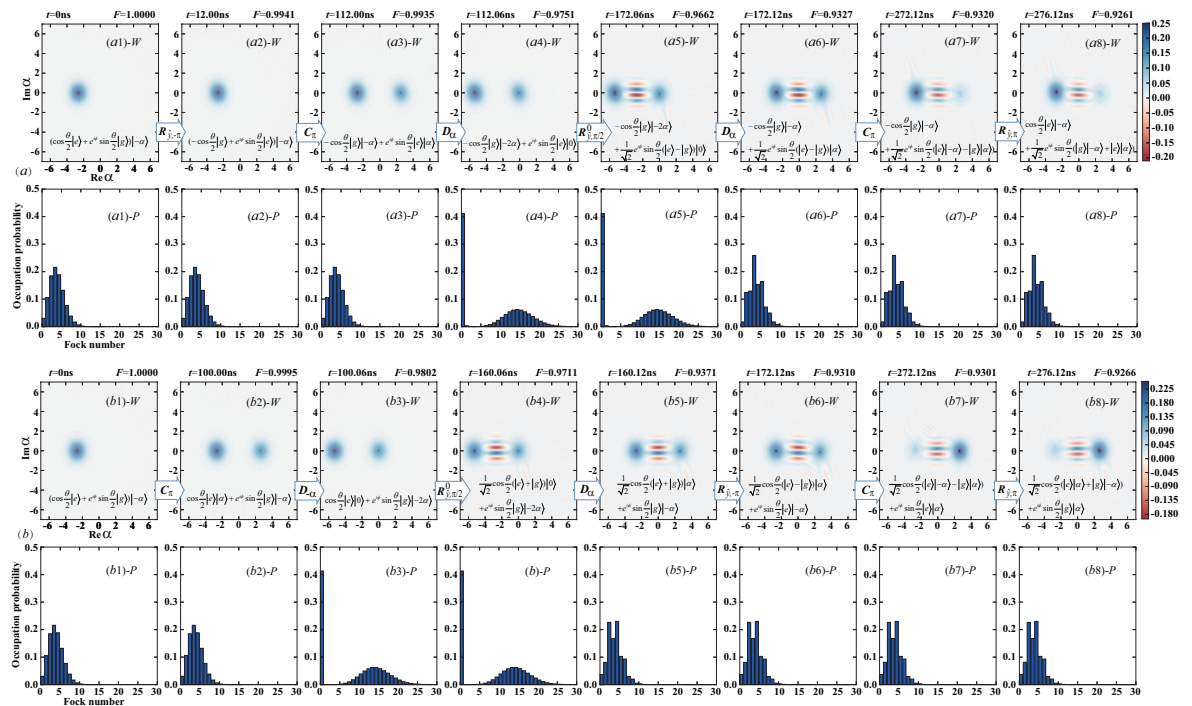

Figure 2: Wigner function and Poisson distribution of the cavity mode after each operation in the cloning transformation sequence as shown in Fig. 1. (a) In the case of the qubit state to be cloned in the northern hemisphere, $\theta=4 \pi / 9$ and $\phi=\pi / 2$. (b) In the case of the qubit state to be cloned in the northern hemisphere, $\theta=5 \pi / 9$ and $\phi=\pi / 2$. The fidelity $F$ to the target state, which is indicated in each panel in respect to Wigner function, and the evolutional time $T$ are shown in the title of each subfigure. 
er tomography and Poisson distribution as shown in Fig. 2, the visualizations of the phase-space probability-distribution-like function for harmonic mode and the occupation probability distribution in the Fock basis can provide additional understanding of cavity mode evolution during the state-dependent cloning. UnWigner function can be negative. The existence of negative quasi-probabilities is generally considered as a signature of nonclassical states [37]. The Wigner functions in Fig. 2 illustrate clear interference fringes, which demonstrate that the evolution is indeed coherent. The Wigner tomography and occupation prob-

rate $\kappa=10^{-4} \chi$, and the qubit relaxation rate $\gamma=\kappa \chi /\left(\omega_{c}-\omega_{q}\right)$. From the Wignlike real probability distribution function, the quasi-probability distribution of ability distribution in Fig. 2 have demonstrated that in which stage during the evolution of cloning process the cavity state is nonclassical. For the stage of $(a 4),(a 5),(b 3)$, or (b4) in Fig. 2 the average photon-number in the cavity is four times than one of the rest stages. This dispersive Hamiltonian as described by Eq. (1) is valid in a low photon-number regime where the dispersive interaction term in Eq. (1) is much greater than higher-order nonlinear terms. From $(a 5) /(b 4)$ to $(a 6) /(b 5)$ the reduced magnitude of the fidelity is larger than the rest. The reduction of the fidelity in the title of each subfigure is mainly due to the photon loss of cavity.

We proposed the scheme for implementation of cloning of qubit states with a priori known value of the polar angle by using a superconducting transmon qubit coupled to a microwave cavity resonator with a highly ideal off-resonant coupling, as shown in Fig. 1. The cloning scheme consists of two logic circuits for the states to be copied in the northern hemisphere and in the southern hemisphere, respectively. From Fig. 3, the fidelity of the cavity mode clone for the present cloning machine based on circuit QED is dependent of the input state vector to be copied on the Bloch sphere. It is worth noting that here the fidelity is related to the final target state of the cavity mode as described in Eq. (6) or Eq. (9). For the present cloning machine, the fidelity of practical output state is smaller than unit. The machine is not the isotropic model because the fidelity value depends on the polar angle and the azimuthal angle of the 

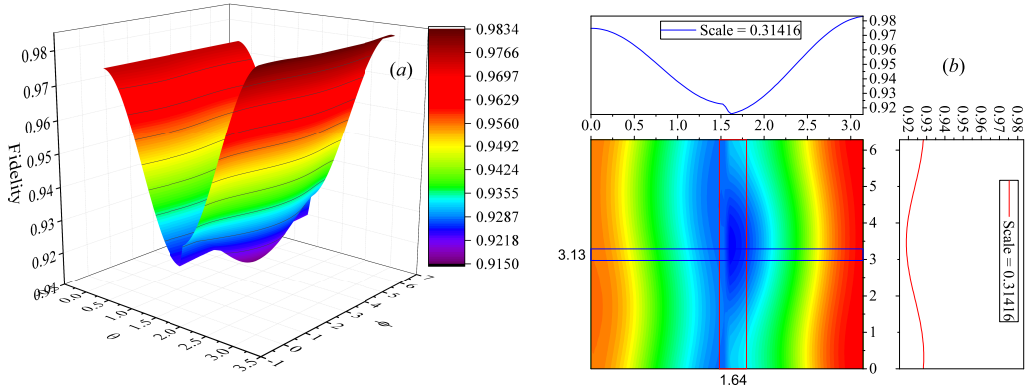

Figure 3: Fidelity of the cavity mode clone as a function of the initial input state parametrized on the logical Bloch sphere by solving numerically the master equation. The fidelity is related to the final target state of the cavity mode. (a) the three-dimensional fidelity as a function of the polar angle and the azimuthal angle; $(b)$ the contour diagram of fidelity as a function of the polar angle and the azimuthal angle.

initial transmon qubit state parametrized on the logical Bloch sphere as shown in Fig. 3. One of the main reasons about the reduction of fidelity is the photon loss of cavity. The other is the quasi-orthogonal computational basis which is used to describe the cavity mode. In addition, the fidelities for the input states from the northern hemisphere and from the southern hemisphere are a little bit asymmetric because we employ two different quantum logic circuits for those states.

We depict the fidelity of the cavity mode clone relative to initial quantum in-

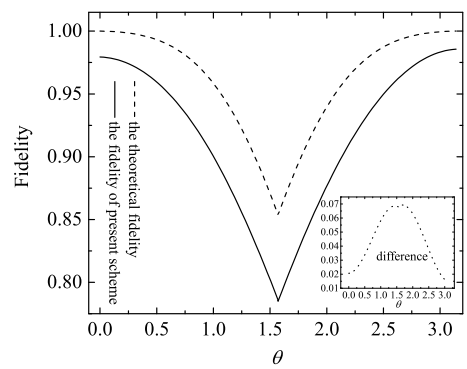

Figure 4: Fidelity of the cavity mode clone as a function of the initial transmon qubit state parametrized on the logical Bloch sphere with the azimuthal angle fixed $\phi=\pi$. 

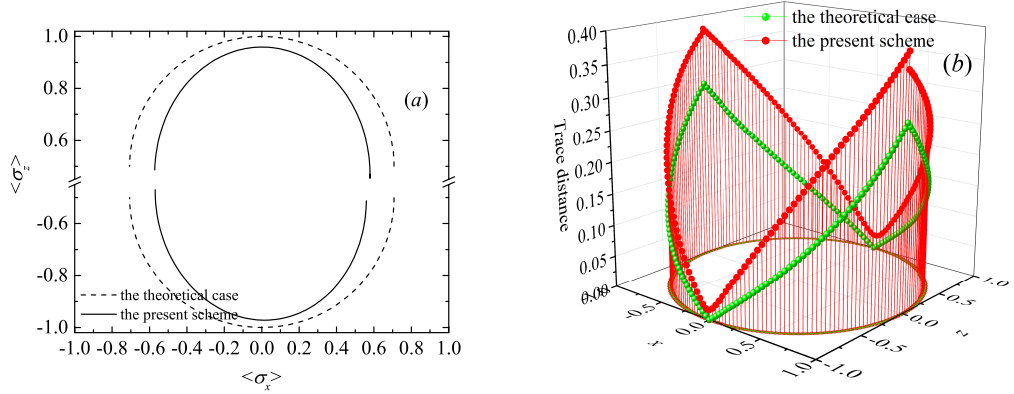

Figure 5: On the condition that the initial input states are restricted to the intersection of the $x$ - $z$ plane with the Bloch sphere, $(a)$ the expectation value $\left\langle\sigma_{z}\right\rangle$ vs. $\left\langle\sigma_{x}\right\rangle$ according to the state of the transmon clone for the theoretical case as described by Eqs. (6 and 9) and for the numerical simulation case of the present scheme by solving the master equation. (b) To see how far the transmon output state is from its input state, we evaluate the trace distances between the input density operator and the theoretical output density operator and between the input density operator and the numerical simulation output density operator.

formation as a function of the polar angle $\theta$ on the logical Bloch sphere about the input state vector while $\phi \equiv \pi$ in Fig. 4 . The dashed line shows the theoretical fidelity as described in Eq. (7) and Eq. (10). The solid line denotes the fidelity of the cavity mode clone in the present cloning scheme by solving numerically the master equation. Although the fidelity of the present scheme is slightly smaller than the theoretical one, the simulation results for the present scheme coincide with the theoretical predictions. This will serve to illustrate that two quantum logic circuits in Fig. 1 by using a superconducting transmon qubit coupled to a microwave cavity enable us to realize efficiently state-dependent cloning transformation. The dotted line represents the difference between the theoretical fidelity and the present scheme one. The difference in the inset of Fig. 4 is consistent with the essence revealed by Fig. 3 .

In the representation of the logical Bloch sphere, the density operator for the initial input state in Eq. (1) can be written as $\rho_{0}=|\varphi\rangle_{q}^{(\text {in) }}\langle\varphi|=\frac{1}{2}(\hat{I}+\vec{r} \cdot \vec{\sigma})$ where $\vec{r}$ denotes the Bloch vector and $\vec{\sigma}$ indicates the vector of Pauli matrices [38]. Then for the quantum cloning machine the reduced density operators for two 
clones are identical $\rho_{q}=\rho_{c}=\rho, \rho=\frac{1}{2}\left(\hat{I}+\vec{r}^{\prime} \cdot \vec{\sigma}\right)$ provided $|0\rangle_{q} \equiv|e\rangle,|1\rangle_{q} \equiv|g\rangle$, $|0\rangle_{c} \equiv|-\alpha\rangle$, and $|1\rangle_{c} \equiv|\alpha\rangle$. Here $\vec{r}^{\prime}=\left(\frac{1}{\sqrt{2}} \sin \theta \cos \phi, \frac{1}{\sqrt{2}} \sin \theta \sin \phi, \frac{1}{2}\left(s_{\theta}+\right.\right.$ $\cos \theta)$ ) according to Eqs. (6 and 9) with $s_{\theta} \equiv \operatorname{sgn}\left(\frac{\pi}{2}-\theta\right)$ [39]. The fidelities of the clones then can be expressed as $F=F_{q}=F_{c}=\frac{1}{2}\left(1+\vec{r} \cdot \vec{r}^{\prime}\right)[38,22]$. It is worth noting that the PCCs in the present scheme are based on the special axisymmetric distribution on the Bloch sphere for the qubits to be cloned, e.g., along the vector $|0\rangle$, that is, along the $z$ axis. Here, the shrinking factor $\vec{r}$. $\vec{r}^{\prime}$, which equals $\frac{1}{\sqrt{2}}+\frac{1}{2} s_{\theta} \cos \theta-\frac{1}{\sqrt{2}}\left(1-\frac{1}{\sqrt{2}}\right) \cos ^{2} \theta$ in the theoretical case, is introduced so as to quantify the qualities of the PCC machines for the restriction of the input set to the different latitudes from the range $[0, \pi]$. For the input state $\rho_{0}$ being a pure state, the length of the Bloch vector $\vec{r}$ is just equal to 1. For the output states after cloning transformation the length of $\vec{r}^{\prime}$ is always smaller than 1. This illustrates that the length of vector $\vec{r}^{\prime}$ shrinks in contrast with one of $\vec{r}$ and the output clones are in the mixed states. From the expression $\vec{r}^{\prime}$ we know the shrinking is not an isotropic compression. For a better view of 245 the negative effect of the cavity decay on the present scheme, we plot the curves about expectation value $\left\langle\sigma_{z}\right\rangle$ vs. $\left\langle\sigma_{x}\right\rangle$ in the quantum state of the transmon qubit clone for the theoretical case as described by Eqs. (6 and 9) and for the simulation case of the present scheme by solving numerically the master equation on the condition that the initial input state is restricted to the intersection of the $x-z$ plane with the Bloch sphere, that is to say, $\sin \phi=0$. From the simulation results of the present scheme in Fig. 5(a), we know that the shrinking degree of the present scheme due to the decay of cavity is slightly larger than one in the theoretical case. It indicates that the states of the actual output clones deviated more severely from the input pure state to be cloned than ones of the theoretical clones. To see how far the output state is from the initial input state, we now evaluate the trace distances [38] between the input density operator and theoretical output density operator and between the input density operator and output density operator by using numerical simulation on the condition that the initial input states are restricted to the intersection of the $x$ - $z$ plane with the Bloch sphere, as depicted by Fig. $5(b)$. The figure of trace distance shows that 
the state of the actual output clone is farther from the input pure state than one of the theoretical clone. From Fig. 5, regardless of the numerical simulation results or the theoretical results, the closer the latitude of the initial input states is to the equator of the Bloch sphere, the smaller the shrinking factor of the for the numerical simulation results are relative to those for the the theoretical results. According to Fig. 5(a) and the expression $F=\frac{1}{2}\left(1+\vec{r} \cdot \vec{r}^{\prime}\right)$, for the actual output clone of the present scheme the fidelity is smaller than one in the theoretical case due to the presence of cavity decay. The curved tendency of simulation results for the present scheme is in accordance with one of the theoretical results. This further corroborates our scheme based on circuit QED can implement efficiently PCC transformation.

\section{Conclusion}

We present a scheme for implementing phase-covariant quantum cloning by using a superconducting transmon qubit coupled to a microwave cavity resonator in the strong dispersive limit of circuit QED. In order to realize the cloning scheme with a priori known expectation value of the Pauli operator $\sigma_{z}$ about the input state, we require four operations: selective rotation operation of the transmon qubit conditioned on the photon number in the cavity, selective phase shift operation of the cavity field conditioned on the qubit state, the unconditional qubit rotation, and the unconditional cavity displacement. We concatenated these operations to design two quantum logic circuits to implement PCC for the input qubit states distributed on the northern hemisphere of the Bloch sphere and on the southern hemisphere, respectively. By solving the master equation, we have accomplished the proposed scheme of PCC with numerical simulation method. We plot the Wigner function and Poisson distribution of the cavity mode after each operation in the cloning transformation sequence according to two logic circuits. The visualizations of the phase-space probability-distribution-like function for harmonic mode and the occupation 
probability distribution in the Fock basis enable us penetrate the evolution process of cavity mode during the PCC transformation. We depict the fidelities of the cavity mode clone, which is related to the final target state of the cavity mode as described in Eq. (6) or Eq. (9), as a function of the initial input state parametrized on the logical Bloch sphere. The cloning machine is not the isotropic model because its output fidelity depends on the polar angle and the azimuthal angle of the initial input state. We compare the fidelity of the cavity mode clone in the present cloning scheme by solving numerically the master equation with one in the theoretical case. In addition, we make a comparison between the numerical simulation result of the shrinking factor in the present cloning scheme and the theoretical result. The result shows the fidelity for the actual output clone of the present scheme by numerical simulation method is slightly smaller than one in the theoretical case. One of the main reasons about the eroded fidelity is the photon loss of cavity. The curved tendency of simulation results is consistent with one of the theoretical results. So our scheme can realize efficiently PCC transformation.

\section{Acknowledgments}

This work was supported by the National Science Foundation of China under Grant No. 11575001 and No. 61275119, and also the Doctoral Foundation of the Ministry of Education of China under Grant No. 20103401110003.

\section{References}

[1] W. K. Wootters, W. H. Zurek, A single quantum cannot be cloned, Nature 299 (5886) (1982) 802-803.

[2] V. Scarani, H. Bechmann-Pasquinucci, N. J. Cerf, M. Dušek, N. Lutkenhaus, M. Peev, The security of practical quantum key distribution, Rev. Mod. Phys. 81 (2009) 1301-1350. 
[3] V. Bužek, M. Hillery, Quantum copying: Beyond the no-cloning theorem, Phys. Rev. A 54 (3) (1996) 1844-1852.

[4] J. Fiurášek, N. J. Cerf, Quantum cloning of a pair of orthogonally polarized photons with linear optics, Phys. Rev. A 77 (2008) 052308.

[5] H. Fan, K. Matsumoto, X. B. Wang, H. Imai, Phase-covariant quantum cloning, J. Phys. A: Math. Gen. 35 (34) (2002) 7415.

[6] D. Bruß, M. Cinchetti, G. Mauro D'Ariano, C. Macchiavello, Phasecovariant quantum cloning, Phys. Rev. A 62 (2000) 012302.

[7] W. H. Zhang, T. Wu, L. Ye, J. L. Dai, Optimal real state cloning in d dimensions, Phys. Rev. A 75 (2007) 044303.

[8] L. M. Duan, G. C. Guo, Probabilistic cloning and identification of linearly independent quantum states, Phys. Rev. Lett. 80 (22) (1998) 4999-5002.

[9] V. Scarani, S. Iblisdir, N. Gisin, A. Acín, Quantum cloning, Rev. Mod. Phys. 77 (4) (2005) 1225-1256.

[10] W. H. Zhang, L. Ye, Optimal asymmetric phase-covariant and real state cloning in d-dimensions, New J. Phys. 9 (9) (2007) 318.

[11] W. H. Zhang, L. B. Yu, L. Ye, Optimal asymmetric phase-covariant quantum cloning, Phys. Lett. A 356 (3) (2006) 195-198.

[12] W. H. Zhang, L. B. Yu, L. Ye, J. L. Dai, Optimal symmetric economical phase-covariant quantum cloning, Phys. Lett. A 360 (6) (2007) 726-730.

[13] V. Bužek, S. L. Braunstein, M. Hillery, D. Bruß, Quantum copying: A network, Phys. Rev. A 56 (5) (1997) 3446-3452.

[14] A. Lamas-Linares, C. Simon, J. C. Howell, D. Bouwmeester, Experimental quantum cloning of single photons, Science 296 (5568) (2002) 712-714. 
[22] H. Fan, Y.-N. Wang, L. Jing, J.-D. Yue, H.-D. Shi, Y.-L. Zhang, L.-Z. Mu, Quantum cloning machines and the applications, Physics Reports 544 (3) (2014) 241-322.

[23] C. Bennett, G. Brassard, Quantum cryptography: Public key distribution

[15] A. Černoch, L. Bartůšková, J. Soubusta, M. Ježek, J. Fiurášek, M. Dušek, Experimental phase-covariant cloning of polarization states of single photons, Phys. Rev. A 74 (2006) 042327.

[16] J. Soubusta, L. Bartůšková, A. Černoch, J. Fiurášek, M. Dušek, Several experimental realizations of symmetric phase-covariant quantum cloners of

[17] E. Nagali, T. De Angelis, F. Sciarrino, F. De Martini, Experimental realization of macroscopic coherence by phase-covariant cloning of a single photon, Phys. Rev. A 76 (2007) 042126.

[18] M. Sabuncu, U. L. Andersen, G. Leuchs, Experimental demonstration of continuous variable cloning with phase-conjugate inputs, Phys. Rev. Lett. 98 (2007) 170503.

[19] H. K. Cummins, C. Jones, A. Furze, N. F. Soffe, M. Mosca, J. M. Peach, J. A. Jones, Approximate quantum cloning with nuclear magnetic resonance, Phys. Rev. Lett. 88 (2002) 187901.

[20] J. F. Du, T. Durt, P. Zou, H. Li, L. Kwek, C. Lai, C. Oh, A. Ekert, Experimental quantum cloning with prior partial information, Phys. Rev. Lett. 94 (4) (2005) 040505.

[21] H. W. Chen, X. Y. Zhou, D. Suter, J. F. Du, Experimental realization of 12 asymmetric phase-covariant quantum cloning, Phys. Rev. A 75 (2007) 012317 .

and coin tossing, in: Proceedings of IEEE International Conference on 
Computers, Systems, and Signal Processing, Proceedings of IEEE International Conference on Computers, Systems, and Signal Processing, IEEE, New York, Bangalore, India, 1984, pp. 175-179.

[24] J. Fiurášek, Extremal equation for optimal completely positive maps, Phys. Rev. A 64 (2001) 062310.

[25] J. Fiurášek, Optical implementations of the optimal phase-covariant quantum cloning machine, Phys. Rev. A 67 (2003) 052314.

[26] H. Fan, K. Matsumoto, X. B. Wang, M. Wadati, Quantum cloning machines for equatorial qubits, Phys. Rev. A 65 (2001) 012304.

[27] R. W. Heeres, B. Vlastakis, E. Holland, S. Krastanov, V. V. Albert, L. Frunzio, L. Jiang, R. J. Schoelkopf, Cavity state manipulation using photon-number selective phase gates, Phys. Rev. Lett. 115 (13) (2015) 137002.

[28] Z. Leghtas, G. Kirchmair, B. Vlastakis, M. H. Devoret, R. J. Schoelkopf, M. Mirrahimi, Deterministic protocol for mapping a qubit to coherent state superpositions in a cavity, Phys. Rev. A 87 (4) (2013) 042315.

[29] B. Vlastakis, G. Kirchmair, Z. Leghtas, S. E. Nigg, L. Frunzio, S. M. Girvin, M. Mirrahimi, M. H. Devoret, R. J. Schoelkopf, Deterministically encoding quantum information using 100-photon schrodinger cat states, Science 342 (6158) (2013) 607-10.

[30] M. Mariantoni, H. Wang, R. C. Bialczak, M. Lenander, E. Lucero, M. Neeley, A. D. O Connell, D. Sank, M. Weides, J. Wenner, T. Yamamoto, Y. Yin, J. Zhao, J. M. Martinis, A. N. Cleland, Photon shell game in threeresonator circuit quantum electrodynamics, Nature Physics 7 (4) (2011) 287-293.

[31] P. J. Leek, M. Baur, J. M. Fink, R. Bianchetti, L. Steffen, S. Filipp, A. Wallraff, Cavity quantum electrodynamics with separate photon storage and qubit readout modes, Phys. Rev. Lett. 104 (2010) 100504. 
[32] H. Paik, D. I. Schuster, L. S. Bishop, G. Kirchmair, G. Catelani, A. P. Sears, B. R. Johnson, M. J. Reagor, L. Frunzio, L. I. Glazman, S. M. Girvin, M. H. Devoret, R. J. Schoelkopf, Observation of high coherence in josephson junction qubits measured in a three-dimensional circuit qed architecture, Phys. Rev. Lett. 107 (24) (2011) 240501.

[33] S. E. Nigg, Deterministic hadamard gate for microwave cat-state qubits in circuit qed, Phys. Rev. A 89 (2014) 022340.

[34] D. I. Schuster, A. A. Houck, J. A. Schreier, A. Wallraff, J. M. Gambetta, A. Blais, L. Frunzio, J. Majer, B. Johnson, M. H. Devoret, S. M. Girvin, R. J. Schoelkopf, Resolving photon number states in a superconducting circuit, Nature 445 (7127) (2007) 515-518.

[35] Z. Leghtas, G. Kirchmair, B. Vlastakis, R. J. Schoelkopf, M. H. Devoret, M. Mirrahimi, Hardware-efficient autonomous quantum memory protection, Phys. Rev. Lett. 111 (12) (2013) 120501.

[36] J. R. Johansson, P. D. Nation, F. Nori, Qutip 2: A python framework for the dynamics of open quantum systems, Comput. Phys. Commun. 184 (4) (2013) 1234-1240.

[37] P. Bertet, A. Auffeves, P. Maioli, S. Osnaghi, T. Meunier, M. Brune, J. Raimond, S. Haroche, Direct measurement of the wigner function of a onephoton fock state in a cavity, Phys. Rev. Lett. 89 (2002) 200402.

[38] M. A. Nielsen, I. L. Chuang, Quantum Computation and Quantum Information, Cambridge University Press, Cambridge, 2000.

[39] K. Bartkiewicz, A. Miranowicz, S. K. Özdemir, Optimal mirror phasecovariant cloning, Phys. Rev. A 80 (2009) 032306. 